\title{
Lab-on-a-Chip Devices and Opportunities for Latin America and the Caribbean
}

\author{
Wilfrido Javier Arteaga Sarmiento, MSc. ${ }^{1}$; Luisa Fernanda Alcalá Zarate, MSc. ${ }^{1}$; Beatriz Lorena Rodríguez \\ Montenegro, MSc. ${ }^{1}$ \\ ${ }^{1}$ Universidad Manuela Beltrán, Bogotá, Colombia, wilfrido.arteaga@docentes.umb.edu.co, luisa.alcala@umb.edu.co, \\ beatriz.rodriguez@umb.edu.co
}

\begin{abstract}
Since about two decades, entrepreneurship has boomed worldwide and our country has not been immune to this movement. With the enactment of Law 1014 of 2006 (Act Entrepreneurship), it's intended that the Colombian educational system training set values that characterize the entrepreneur and encouraging greater entrepreneurial training both in society and in all programs of education by creating a set of normative principles and state policies that articulate to spread awareness promotion of entrepreneurship and business creation. The project has a wide range of topics that may be amenable to analysis, such as: social entrepreneurship, family businesses, funding business plans, characteristics of entrepreneurs, among others. This article presents a much more rigorous study of the characteristics that scholars have our program of industrial engineering and framing your profile as entrepreneurs. The characteristics that were considered for this study are: Leadership, Creativity and Focus on results.
\end{abstract}

Keywords- Entrepreneurship, Entrepreneur Profile, Character, Leadership, creativity, results orientation

Digital Object Identifier (DOI): http://dx.doi.org/10.18687/LACCEI2015.1.1.194

ISBN: 13 978-0-9822896-8-6

ISSN: 2414-6668

$1^{\text {th }}$ LACCEI Annual International Conference: “Engineering Education Facing the Grand Challenges, What Are We Doing?” July 29-31, 2015, Santo Domingo, Dominican Republic

DOI: http://dx.doi.org/10.18687/LACCEI2015.1.1.194 


\title{
Exploración del Perfil Emprendedor del Estudiante de Ingeniería Industrial de la Universidad Manuela Beltrán
}

\author{
MSc. Ing. Wilfrido Javier Arteaga Sarmiento \\ Grupo de Investigación Gestión Gerencial - Docente de Planta \\ Universidad Manuela Beltrán, Bogotá, Colombia, wilfrido.arteaga@docentes.umb.edu.co \\ MSc. Ing. Luisa Fernanda Alcalá Zarate \\ Grupo de Investigación Gestión Gerencial - Coordinadora de Investigación \\ Universidad Manuela Beltrán, Bogotá, Colombia, luisa.alcala@umb.edu.co \\ MSc. Ing. Beatriz Lorena Rodríguez Montenegro \\ Directora de Programa de Ingeniería Industrial \\ Universidad Manuela Beltrán, Bogotá, Colombia, beatriz.rodriguez@umb.edu.co
}

\begin{abstract}
Since about two decades, entrepreneurship has boomed worldwide and our country has not been immune to this movement. With the enactment of Law 1014 of 2006 (Act Entrepreneurship), it's intended that the Colombian educational system training set values that characterize the entrepreneur and encouraging greater entrepreneurial training both in society and in all programs of education by creating a set of normative principles and state policies that articulate to spread awareness promotion of entrepreneurship and business creation. The project has a wide range of topics that may be amenable to analysis, such as: social entrepreneurship, family businesses, funding business plans, characteristics of entrepreneurs, among others. This article presents a much more rigorous study of the characteristics that scholars have our program of industrial engineering and framing your profile as entrepreneurs. The characteristics that were considered for this study are: Leadership, Creativity and Focus on results.
\end{abstract}

Keywords- Entrepreneurship, Entrepreneur Profile, Character, Leadership, creativity, results orientation

Resumen-Desde hace aproximadamente dos décadas, el emprendimiento ha tenido un gran auge a nivel mundial y nuestro país no ha sido ajeno a este movimiento. Con la sanción de la ley 1014 de 2006 (Ley de Emprendimiento), se busca que el sistema educativo colombiano establezca la formación en valores que caracterizan la figura del emprendedor y el fomento de una mayor formación empresarial tanto en la sociedad como en todos los programas de enseñanza, mediante la creación de un conjunto de principios normativos y políticas estatales que se articulan para generar una conciencia de promoción del emprendimiento y creación de empresas. El emprendimiento tiene una amplia gama de tópicos que pueden ser susceptible de análisis, tales como: el emprendimiento social, las famiempresas, la financiación de planes de negocio, las características del emprendedor, entre otros aspectos. Este artículo presenta un estudio mucho más riguroso sobre las características que poseen nuestros estudiosos del programa de ingeniería industrial y que enmarcan su perfil como emprendedores. Las características que se tuvieron en cuenta para el presente estudio son: Liderazgo, Creatividad y Orientación a resultados.
Palabras Claves-Emprendimiento, Perfil Emprendedor, Caracterización, Liderazgo, Creatividad, Orientación a resultados

\section{INTRODUCCIÓN}

El emprendimiento ha sido estudiado desde diversas áreas del conocimiento como: la economía, la psicología, la sociología y la antropología. En un principio, la economía se encargó de estudiarlo buscando la relación del mismo con el desarrollo desde un punto de vista puramente económico. Posteriormente, la psicología, la sociología y la antropología desarrollaron aportes que se encargaron del estudio del fenómeno desde un punto de vista social (Guzmán-Trujillo, 2008), este aporte es relevante ya que nos permitió entender que los valores, las creencias, las ideologías, los hábitos, las costumbres y las normas, que comparten los individuos en una organización y que surgen de la interrelación social, generan patrones de comportamiento que establecen una identidad entre sus miembros, que son definitivas en su capacidad de generar bienes y servicios de una forma creativa, metódica, ética, responsable y efectiva.

Todo esto llevado a cabo a través de un liderazgo equilibrado y justo y de una orientación a resultados que genere valor y que beneficie a la empresa, a la economía y a la sociedad en general. Con la situación económica mundial, miles de personas al quedarse desempleadas y miles de nuevos egresados al no encontrar trabajo, recurren a tratar de comenzar su propio negocio, realmente con poca preparación y conocimiento acerca de cómo funcionan los negocios (Audretsch, 2005).

La realidad es que aproximadamente el $90 \%$ de las empresas que se crean, cierran en los primeros años de vida y las que sobreviven necesitan mucho tiempo para alcanzar una rentabilidad aceptable (Lussier, 2005). 
En Colombia, se puede decir que el proceso para formalizar, por vía gubernamental, todo el tema relacionado con emprendimiento, pasó por muchas etapas en la que finalmente, bajo el gobierno del presidente Álvaro Uribe Vélez, se aprobó la Ley 1014 de 2006 más conocida como Ley de Emprendimiento. Esta ley generó elementos complementarios que permiten incentivar el emprendimiento y estimuló un ambiente ideal para la creación de empresas, así mismo brindó las herramientas para el fomento de un espíritu emprendedor a través de la implementación de cátedras en todas las instituciones de educación formal y no formal del país.

La educación del emprendimiento en las últimas décadas ha cobrado importancia gracias a que plantea el emprendimiento como una disciplina susceptible de ser enseñada y aprendida, (Kuratko, 2003). El número de cursos cuyo tópico central es el emprendimiento, ha aumentado de manera considerable en los diferentes programas sobre preparación empresarial en el contexto nacional e internacional. De igual forma, bajo este enfoque, se considera la formación en las capacidades, habilidades y conocimientos que permitan desarrollar un potencial emprendedor en los individuos, las cuales han surgido de la observación de emprendedores empresarios con diferentes niveles de éxito

El objetivo de este trabajo es realizar la caracterización del perfil emprendedor del estudiante del programa de ingeniería industrial de la universidad Manuela Beltrán en aspectos de liderazgo, creatividad y orientación a resultados.

El presente artículo se divide en cinco partes: la primera es la introducción, la segunda corresponde a la descripción del emprendimiento en Colombia, la tercera a la metodología y los resultados del estudio, la cuarta a la discusión y la quinta las conclusiones

\section{EMPRENDIMIENTO EN COLOMBIA}

Cuando se habla de emprendimiento en Colombia, muchas veces se asocia con instituciones que promueven únicamente la creación de un negocio, bajo la aprobación de un comité que analiza la viabilidad del mismo y aporta un capital semilla con el fin de impulsarlas, pero en realidad es mucho más que eso. El emprendimiento es un tema que viene siendo tratado alrededor de 30 años en Colombia, pero regulado como ley hace trece años, basando la formación en competencias para el emprendimiento a entidades educativas, empresa y estado, con el fin de fortalecer las organizaciones existentes y crear nuevas empresas competitivas e innovadoras en el mercado actual.

Dentro de la evolución del emprendimientos en Colombia, hubo personalidades, leyes y entidades involucradas en busca de que esto germinara hasta convertirse en lo que es hoy, una Política Nacional.
A continuación se relación el surgir del emprendimiento en Colombia, el cual describe entidades y personalidad que intervinieron en el crecimiento del mismo:

1. Unas de las entidades pionera en buscar preparar empresarios para que crearan nuevas empresas, fue el SENA, Servicio Nacional de Aprendizaje, el cual se encuentra adscrito al Ministerio de trabajo de Colombia y ofrece formación gratuita con programas técnicos, tecnológicos enfocados al desarrollo del país, buscando engrandecer las actividades de las empresa, con el fin de que sean más competidoras en el entorno que se encuentra. (SENA, 1993)

2. A finales de Gobierno del presidente Virgilio Barco, se aprobó la Ley 29 por la cual se dictan disposiciones para el fomento de la investigación científica y el desarrollo tecnológico (Congreso de la República de Colombia, 1990)

3. La Constitución Política de 1991, que se promulgó durante el gobierno de César Gaviria, se introdujeron una serie de figuras jurídicas relacionadas con la apertura del mercado, el llamado neoliberalismo, que marca el derrotero económico del país. En este año se reglamentó la Ley de Ciencia y Tecnología.

4. Informe Misión de Sabios; Colombia al filo de la Oportunidad documento propuesto por el Presidente Gaviria, en donde se dan recomendaciones acerca de las organizaciones, la educación, la ciencia y la tecnología.

5. Aprobación de la ley 344 del 1996 (Congreso de la República de Colombia, 1996) donde se reglamenta destinar recursos para el desarrollo tecnológico.

6. Aprobación de la Ley 375 de la Juventud (Congreso de la República de Colombia, 1997). Esta ley pretende promover la formación integral del joven para que participe activamente en todas las áreas socioeconómicas con el fin de generar un impacto en la nación.

7. Aprobación de la Ley 590 de 2000, Impulsar el desarrollo de la micro, pequeña y mediana empresa colombiana. (Congreso de la República de Colombia, 2000).

8. Documento aprobado, "Política para el fomento del espíritu empresarial y la creación de empresas" (Ministerio de Comercio, Industria y Turismo, 2000)

9. Aprobación de la Ley 789 de 2002, Creación FONDO EMPRENDER. (Congreso de la República de Colombia, 2002).

10. La Ley 590 de 2000 modificada por Ley 905 de 2004 (Congreso de la República de Colombia, 2004).

11. Ley 1014 de 2006, Fomento a la cultura de emprendimiento. (Congreso de la República de Colombia, 2006). 
12. Ley 1151 de 2007, "Plan Nacional de Desarrollo 2006-2010 (Congreso de la República de Colombia, 2006).

13. Decreto 4466 de 2006, por el cual se reglamenta el artículo 22 de la Ley 1014 de 2006, sobre constitución de nuevas empresas.

14. Documento CONPES 3297 del 26 de julio de 2004, que define la Agenda Interna para la Productividad y Competitividad. (DNP, 2004)

15. Documento CONPES 3424 del 16 de mayo de 2006, denominada la Banca de las Oportunidades, el cual busca promover el acceso al crédito y a los demás servicios financieros buscando la equidad social en Colombia. (DNP, 2006)

16. Aprobación de la Ley 1258 de 2008 (Congreso de la República de Colombia, 2008); se crean las sociedades anónimas simplificadas

17. Documento CONPES 3439 del 14 de agosto de 2006, que crea el Sistema Administrativo Nacional de Competitividad y como su instancia coordinadora la Comisión Nacional de Competitividad.

(El Decreto 1475 de mayo de 2008 incluye al MCIT en la Secretaria Técnica y define que MCIT y Confecámaras coordinarán las Comisiones Regionales de Competitividad). (DNP, 2006)

18. Documento CONPES 3484 del 13 de agosto de 2007, habla sobre la política nacional para la transformación productiva y la mejora sostenible de la productividad y competitividad de las Mipymes. (DNP, 2007)

19. Documento CONPES 3527 del 23 de junio de 2008, sobre la Política Nacional de Competitividad y Productividad. (DNP, 2008)

Dentro de todos los antecedentes mencionados, se puede observar que a partir de la aprobación de la Ley de emprendimiento, Ley 1014 del 2006, Colombia viene con tendencias crecientes, el cual viene visibilizándose en los demás países, abriendo puertas a los empresarios presentes y futuros.

GRAFICA I

COMPORTAMIENTO ACTIVIDAD EMPRENDEDORA EN COLOMBIA 2006 - 2011 (Fuente: Informe GEM (2006,2007, 2008, 2009, 2010, 2011)

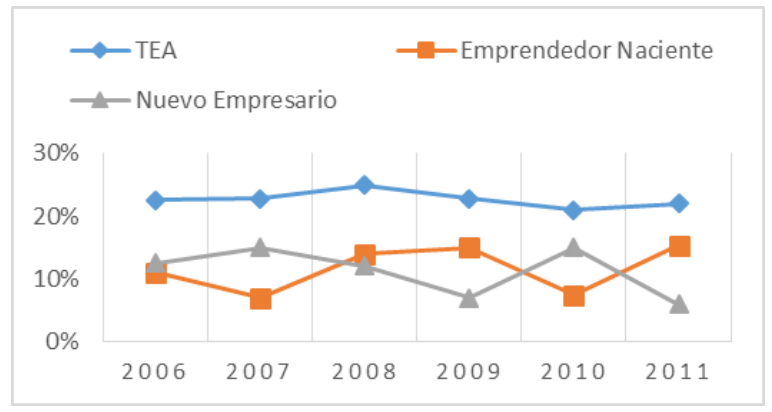

Uno de los actores principales en esta ley es la educación, donde ha sido identificada como pieza clave dentro de este proceso, ya que el objetivo de este sistema es fomentar la cultura desde los primero años de formación. Según el Ministerio de Educación Nacional en la Guía No 39, menciona que el promover el emprendimiento, se constituye en una oportunidad para que los estudiantes apliquen sus actitudes emprendedoras y encaminen sus esfuerzos a la creación de empresas sostenibles, productivas con aplicaciones tecnológicas entre otras. (Ministerio de Educación Nacional, 2012), por lo tanto, la Universidad Manuela Beltrán, como institución que promueve la investigación, la innovación y el emprendimiento no solo de los estudiantes, sino en toda la organización, ha buscado no quedarse atrás con respecto a esta política. Unas de las estrategias implementadas son asignaturas que continúen esa línea de formación de manera transversal a todos los programas.

El programa de Ingeniería industrial en el año 2012, bajo la aprobación de la Directivas de la Universidad, propuso un Centro de emprendimiento, llamado CREAR; este escenario es una manera de enseñar, re direccionar y apoyar aquellos proyectos viables propuestos por los estudiantes, egresados y docentes, con el fin de fomentar y desarrollar la cultura del emprendimiento y la creación de empresas, enmarcada en la ley 1014 de 2006, desarrollando y acompañando procesos de generación de ideas de negocios, estructurando y fortaleciendo planes de negocios y acompañando en la creación y puesta en marcha de nuevas empresas, como contribución a la generación de empleo y participación en la economía de nuestro país.

Estos antecedentes dan cuenta del amplio interés por entender y predecir las condiciones individuales que facilitan o dificultan el comportamiento emprendedor, entendidas como actitudes, aptitudes, habilidades y rasgos, entre otras. Este documento tiene por objetivo ser un punto de referencia para describir y analizar características insertas en integrantes de la comunidad universitaria de la Universidad Manuela Beltrán de Colombia, Sede Bogotá, que pueden incidir en el desarrollo de nuevos proyectos emprendedores. Lo anterior con base en los resultados de la coherencia interna entre las variables propuestas para hallar un potencial perfil emprendedor.

\section{Metodología Y RESUltados}

El centro de emprendimiento de la Universidad Manuela Beltrán, CREAR, fomenta por medio de la motivación, capacitación, asesoría, consultoría e investigación, el espíritu emprendedor, característica de nuestra Universidad, con el fin de generar una dinámica en nuestro entorno hacia la creación de empresas, desarrollando proyectos para apoyar el fortalecimiento empresarial e institucional. 
De esta manera y a partir de los elementos encontrados en la revisión bibliográfica y en las experiencias obtenidas por el equipo humano del centro de Emprendimiento y la Dirección del Programa de Ingeniería Industrial, se construyó el instrumento de recolección de información conformado por 17 ítems para evaluar tres dimensiones: Liderazgo (LI), Creatividad (CR) y Orientación a Resultados (OR); las cuales se han definido de la siguiente manera:

Liderazgo: En la literatura, el liderazgo ejercido para el emprendimiento, se relaciona con las habilidades para motivar y hacer que un equipo alcanzase una meta en común. Para Cardoso (2003), el líder está en disposición de identificar necesidades y desarrollar planes de acción para solventar las situaciones, anticipa con claridad el problema e influencia a otros para que se dirijan a resolver la necesidad en común, más que a solo alcanzar una meta externa. El líder está atento a la negociación y la asume como una forma de interacción social para la solución de conflictos e intereses, donde se buscan varias estrategias posibles para la solución, y considera que el acuerdo es mejor a la ausencia del mismo, así como la intencionalidad en la posibilidad de alcanzarlo (Páez - García, 2007)

Creatividad: Zhao y Seibert (2006) examinan la relación entre personalidad y las condiciones de emprendimiento, y categorizan las variables de personalidad de acuerdo con el modelo de los cinco factores. Una de esas variables es la apertura a nuevas experiencias que caracterizaría a una persona como creativa, innovadora, imaginativa y reflexiva. Para Csickzentmihalyi (1998), la creatividad se plantea en una triada de campo (lugar o disciplina), persona (quien realiza el acto creativo) y dominio (grupo del conocimiento). Y la define como "el estado de conciencia que permite generar una red de relaciones para identificar, plantear, resolver problemas de manera relevante y divergente." En virtud de lo anterior, la creatividad, desde el emprendimiento, puede entenderse como la capacidad de explorar nuevas e interesantes posibilidades y hacer algo al respecto, sabiendo que está acción producirá cambios importantes en el entorno del emprendedor.

$>$ Orientación a resultados: Spencer (1993) define la orientación al logro como el esfuerzo que hacen las personas como individuos y el equipo de trabajo como unidad, por cumplir con los objetivos planteados en un proyecto, dentro de las expectativas esperadas. En este sentido, puede entenderse como la como la capacidad del emprendedor para actuar con velocidad, sentido de urgencia y diligencia en el logro de los objetivos que le resultan desafiantes. Este esfuerzo es un reflejo de una actitud personal y de grupo orientada a cumplir con los compromisos.

Los diferentes estudios que se han realizado sobre la personalidad y el perfil emprendedor se dirigen principalmente a explorar y describir los rasgos y características de personalidad que permitan predecir comportamientos emprendedores. Según Hornaday Uriarte y Martín (2007), la investigación sobre las características del individuo emprendedor apunta a tres cuestiones principales:

$>$ La existencia de característica de índoles psicológicas, sociales, hereditarias, ambientales, educacionales, o experimentales que diferencien al emprendedor con éxito de otros grupos humanos.

$>$ De ser así, si es posible que esas características existan previamente al comportamiento emprendedor.

> Determinar la posibilidad de evaluarlas y anticiparlas al proceso de la creación de empresa.

El instrumento de recolección de información se aplicó a una muestra conformada por 96 estudiantes del programa de ingeniería industrial de la Universidad Manuela Beltrán, 50\% hombres y 50\% mujeres en el primer trimestre del 2015. La confiabilidad del presente estudio está dada por los siguientes parámetros: $93 \%$ de confianza y $7 \%$ de error, determinados a partir del universo total de los estudiosos del programa.

Para determinar el grado de cumplimiento de cada una de las características estudiadas, los investigadores del presente artículo diseñaron una escala de gradación porcentual. Igualmente se establecieron los rangos de porcentaje de logro y la valoración cualitativa, por medio de la cual se podrán establecer, a futuro, acciones que lleven al fortalecimiento de las características emprendedoras de los estudiosos del programa de Ingeniería Industrial de la universidad Manuela Beltrán.

TABLA I

GRADACIÓN: CARACTERÍSTICA LIDERAZGO

\begin{tabular}{|c|l|l|}
\hline $\begin{array}{c}\text { Grado de } \\
\text { Cumplimiento }\end{array}$ & $\begin{array}{l}\text { Codificación } \\
\text { Cuantitativa }\end{array}$ & Valoración Cualitativa \\
\hline 5 & $90 \%-100 \%$ & Se cumple plenamente. \\
& & Se entiende como la \\
& fortaleza de los \\
& estudiosos en la \\
& Capacidad para fijar \\
& objetivos, realizar el \\
& seguimiento y orientar y \\
& motivar a los demás para \\
& la consecución de los \\
& mismos creando un \\
& ambiente basado en la \\
& confianza mutua y el \\
& desarrollo \\
& personal/profesional \\
\hline
\end{tabular}

13 $^{\text {th }}$ LACCEI Annual International Conference: "Engineering Education Facing the Grand Challenges, What Are We Doing?" July 29-31, 2015, Santo Domingo, Dominican Republic 


\begin{tabular}{|c|c|c|}
\hline 4 & $75 \%-89 \%$ & $\begin{array}{l}\text { Se cumple en alto grado. } \\
\text { Son fortalezas que posee } \\
\text { el estudioso y que deben } \\
\text { tender al mejoramiento } \\
\text { pleno, orientadas a } \\
\text { impactar profundamente } \\
\text { en la motivación } \\
\text { personal y de su grupo. } \\
\text { Tiene una definición } \\
\text { clara de su ser y su } \\
\text { quehacer. Presenta } \\
\text { algunas oportunidades } \\
\text { de mejoramiento para } \\
\text { alcanzar el máximo } \\
\text { nivel }\end{array}$ \\
\hline 3 & $50 \%-74 \%$ & $\begin{array}{l}\text { Se cumple } \\
\text { aceptablemente. } \\
\text { Presenta fortalezas y } \\
\text { debilidades que son } \\
\text { susceptibles de mejorar, } \\
\text { no se alcanzan los } \\
\text { ideales de un excelente } \\
\text { liderazgo }\end{array}$ \\
\hline 2 & $26 \%-49 \%$ & $\begin{array}{l}\text { Se cumple } \\
\text { insatisfactoriamente. No } \\
\text { responde a los ideales de } \\
\text { un liderazgo eficiente, } \\
\text { requiere atención porque } \\
\text { deber mejorarse } \\
\text { notablemente los rasgos } \\
\text { que definen su liderazgo }\end{array}$ \\
\hline 1 & $1 \%-25 \%$ & $\begin{array}{l}\text { No se cumple. Le falta } \\
\text { interés y voluntad para } \\
\text { desarrollar habilidades } \\
\text { de líder. Presenta más } \\
\text { debilidades } \\
\text { fortalezas }\end{array}$ \\
\hline
\end{tabular}

Una vez aplicadas las encuestas se determinó el nivel de cumplimiento de la característica liderazgo y así se determinó las fortalezas de los estudiosos del programa. Estos hallazgos serán el punto de partida para tener la caracterización del perfil emprendedor de nuestro estudioso y así establecer estrategias para la consolidación de su liderazgo en la sociedad local, regional y nacional:

TABLA II

RESULTADOS: CARACTERÍSTICA LIDERAZGO

\begin{tabular}{|l|c|c|}
\hline \multicolumn{1}{|c|}{ Característica Liderazgo } & \multicolumn{2}{c|}{ Resultados 2015 } \\
\cline { 2 - 3 } & \% Total & $\begin{array}{c}\text { Grado de } \\
\text { Cumplimiento }\end{array}$ \\
\hline $\begin{array}{l}\text { ¿Te propones alcanzar los objetivos } \\
\text { (Profesionales/Personales) que te has trazado } \\
\text { en tu proyecto de vida? }\end{array}$ & 96 & $\begin{array}{c}\text { Se cumple } \\
\text { plenamente }\end{array}$ \\
\hline $\begin{array}{l}\text { ¿Eres constante con las iniciativas que } \\
\text { emprendes? }\end{array}$ & 94 & $\begin{array}{c}\text { Se cumple } \\
\text { plenamente }\end{array}$ \\
\hline $\begin{array}{l}\text { ¿Te formulas estrategias personales y acciones } \\
\text { pertinentes que suponen te agreguen valor } \\
\text { tanto en lo personal como en tu desarrollo } \\
\text { profesional? }\end{array}$ & 85 & $\begin{array}{c}\text { Se cumple en alto } \\
\text { grado }\end{array}$ \\
\hline $\begin{array}{l}\text { ¿Tomas parte de algo o te comprometes en un } \\
\text { asunto o tarea personalmente? }\end{array}$ & 96 & $\begin{array}{c}\text { Se cumple } \\
\text { plenamente }\end{array}$ \\
\hline $\begin{array}{l}\text { ¿Tienes influencia activa en cualquier } \\
\text { acontecimiento? }\end{array}$ & 76 & $\begin{array}{c}\text { Se cumple en alto } \\
\text { grado }\end{array}$ \\
\hline Total Característica & 95 & $\begin{array}{c}\text { Se cumple } \\
\text { plenamente }\end{array}$ \\
\hline ¿Tienes confianza en ti mismo? & 91 & $\begin{array}{c}\text { Se cumple } \\
\text { plenamente }\end{array}$ \\
\hline $\begin{array}{l}\text { ¿Tiene, ha tenido o piensa desarrollar alguna } \\
\text { idea de negocio? }\end{array}$ & 98 & $\begin{array}{c}\text { Se cumple } \\
\text { plenamente }\end{array}$ \\
\hline
\end{tabular}

De acuerdo al análisis anterior, se concluye que la característica de liderazgo se cumple plenamente con un promedio de $91 \%$, denotando la capacidad del estudioso del programa de Ingeniería Industrial para fijar objetivos, realizar el seguimiento y orientar y motivar a los demás para la consecución de los mismos creando un ambiente basado en la confianza mutua y el desarrollo tanto personal como profesional

TABLA III

GRADACIÓN: CARACTERÍSTICA CREATIVIDAD

\begin{tabular}{|c|c|c|}
\hline $\begin{array}{c}\text { Grado de } \\
\text { Cumplimiento }\end{array}$ & $\begin{array}{l}\text { Codificación } \\
\text { Cuantitativa } \\
\end{array}$ & Valoración Cualitativa \\
\hline 5 & $90 \%-100 \%$ & $\begin{array}{l}\text { Se cumple plenamente. } \\
\text { Se entiende como la } \\
\text { fortaleza de los } \\
\text { estudiosos en la } \\
\text { Capacidad para idear } \\
\text { planteamientos } \\
\text { soluciones nuevas y } \\
\text { diferentes para resolver } \\
\text { problemas o situaciones } \\
\text { requeridas por los } \\
\text { clientes o el segmento de } \\
\text { la economía en el que se } \\
\text { actúe }\end{array}$ \\
\hline 4 & $75 \%-89 \%$ & $\begin{array}{l}\text { Se cumple en alto grado. } \\
\text { Son fortalezas que posee } \\
\text { el estudioso y que deben } \\
\text { tender al mejoramiento } \\
\text { pleno, orientadas a } \\
\text { impactar profundamente } \\
\text { en innovación y } \\
\text { asertividad r para } \\
\text { proponer nuevas } \\
\text { alternativas de solución } \\
\text { a los diferentes } \\
\text { problemas que puedan } \\
\text { surgir. Tiene una } \\
\text { definición clara de su ser }\end{array}$ \\
\hline
\end{tabular}

13 $^{\text {th }}$ LACCEI Annual International Conference: "Engineering Education Facing the Grand Challenges, What Are We Doing?" July 29-31, 2015, Santo Domingo, Dominican Republic 


\begin{tabular}{|c|c|c|}
\hline & & $\begin{array}{l}\text { y su quehacer. Presenta } \\
\text { algunas oportunidades } \\
\text { de mejoramiento para } \\
\text { alcanzar el máximo } \\
\text { nivel }\end{array}$ \\
\hline 3 & $50 \%-74 \%$ & $\begin{array}{l}\text { Se cumple } \\
\text { aceptablemente. } \\
\text { Presenta fortalezas y } \\
\text { debilidades que son } \\
\text { susceptibles de mejorar, } \\
\text { no se alcanzan los } \\
\text { ideales que permitan } \\
\text { demostrar, a través de la } \\
\text { práctica, altos niveles de } \\
\text { creatividad } \\
\text { innovación. }\end{array}$ \\
\hline 2 & $26 \%-49 \%$ & $\begin{array}{l}\text { Se cumple } \\
\text { insatisfactoriamente. No } \\
\text { responde a los ideales de } \\
\text { un creativo e innovador } \\
\text { eficiente, requiere } \\
\text { atención porque debe } \\
\text { mejorarse notablemente } \\
\text { los rasgos que definen } \\
\text { su creatividad }\end{array}$ \\
\hline 1 & $1 \%-25 \%$ & $\begin{array}{l}\text { No se cumple. Le falta } \\
\text { interés y voluntad para } \\
\text { desarrollar habilidades } \\
\text { creativas. Presenta más } \\
\text { debilidades que } \\
\text { fortalezas }\end{array}$ \\
\hline
\end{tabular}

Una vez aplicadas las encuestas se determinó el nivel de cumplimiento de la característica creatividad y así se determinó las fortalezas de los estudiosos del programa. Estos resultados permitirán el planteamiento de unas recomendaciones y/o acciones tendientes al fortalecimiento del perfil emprendedor del estudioso del programa de Ingeniería Industrial, mediante la construcción de un modelo de extensión y proyección social como soporte del fomento de la cultura empresarial, la creación y puesta en marcha de nuevas empresas para el desarrollo económico y social del país:

TABLA IV

RESULTADOS: CREATIVIDAD

\begin{tabular}{|c|c|c|}
\hline \multirow[b]{2}{*}{ Característica Creatividad } & \multicolumn{2}{|c|}{ Resultados 2015} \\
\hline & $\%$ Total & $\begin{array}{c}\text { Grado de } \\
\text { Cumplimiento }\end{array}$ \\
\hline $\begin{array}{l}\text { Los entornos inciertos, sin reglas, sin límites, } \\
\text { son para ti un reto intelectual, te resultan } \\
\text { especialmente motivadores y te permiten } \\
\text { disfrutar aprendiendo e innovando }\end{array}$ & 75 & $\begin{array}{l}\text { Se cumple en alto } \\
\text { grado }\end{array}$ \\
\hline $\begin{array}{l}\text { ¿Sacas provecho de cualquier situación } \\
\text { novedosa o de cambio? }\end{array}$ & 85 & $\begin{array}{l}\text { Se cumple en alto } \\
\text { grado }\end{array}$ \\
\hline $\begin{array}{l}\text { ¿Eres capaz de generar respuestas innovadoras } \\
\text { a situaciones complejas que se presentan? }\end{array}$ & 84 & $\begin{array}{c}\text { Se cumple en alto } \\
\text { grado }\end{array}$ \\
\hline $\begin{array}{l}\text { ¿Eres capaz de producir soluciones originales o } \\
\text { poco habituales a un problema? }\end{array}$ & 84 & $\begin{array}{l}\text { Se cumple en alto } \\
\text { grado }\end{array}$ \\
\hline Total Característica & 82 & $\begin{array}{l}\text { Se cumple en alto } \\
\text { grado }\end{array}$ \\
\hline
\end{tabular}

De acuerdo al análisis anterior, se concluye que la característica de creatividad se cumple en alto grado con un promedio de $82 \%$, denotando las fortalezas que posee el estudioso del programa de Ingeniería Industrial y que deben tender al mejoramiento pleno, orientadas a impactar profundamente en innovación y asertividad para proponer nuevas alternativas de solución a los diferentes problemas que puedan surgir

TABLA V

GRADACIÓN: CARACTERÍSTICA ORIENTACIÓN A RESULTADOS

\begin{tabular}{|c|c|c|}
\hline $\begin{array}{c}\text { Grado de } \\
\text { Cumplimiento }\end{array}$ & $\begin{array}{l}\text { Codificación } \\
\text { Cuantitativa }\end{array}$ & Valoración Cualitativa \\
\hline 5 & $90 \%-100 \%$ & $\begin{array}{l}\text { Se cumple plenamente. } \\
\text { Se entiende como la } \\
\text { fortaleza de los } \\
\text { estudiosos } \\
\text { Predisposición a actuar } \\
\text { con un claro interés por } \\
\text { conseguir los objetivos } \\
\text { marcados, fijando metas } \\
\text { desafiantes por encima } \\
\text { de los estándares, } \\
\text { mejorando y } \\
\text { manteniendo altos } \\
\text { niveles de rendimiento }\end{array}$ \\
\hline 4 & $75 \%-89 \%$ & $\begin{array}{l}\text { Se cumple en alto grado. } \\
\text { Son fortalezas que posee } \\
\text { el estudioso y que deben } \\
\text { tender al mejoramiento } \\
\text { pleno, orientadas a } \\
\text { impactar profundamente } \\
\text { su propio desafío para la } \\
\text { consecución r de } \\
\text { objetivos de interés } \\
\text { propio y general. Tiene } \\
\text { una definición clara de } \\
\text { su ser y su quehacer. } \\
\text { Presenta } \\
\text { oportunidades algunas } \\
\text { mejoramiento de } \\
\text { alcanzar el mara } \\
\text { nivel rimo }\end{array}$ \\
\hline 3 & $50 \%-74 \%$ & $\begin{array}{l}\text { Se cumple } \\
\text { aceptablemente. } \\
\text { Presenta fortalezas y } \\
\text { debilidades que son } \\
\text { susceptibles de mejorar, } \\
\text { no se alcanzan los } \\
\text { ideales que permitan } \\
\text { demostrar, a través de la } \\
\text { práctica, constancia y } \\
\text { perseverancia para el } \\
\text { logro de las metas. }\end{array}$ \\
\hline 2 & $26 \%-49 \%$ & $\begin{array}{l}\text { Se cumple } \\
\text { insatisfactoriamente. No } \\
\text { responde a los ideales de } \\
\text { un consolidador de } \\
\text { sueños, requiere } \\
\text { atención porque debe } \\
\text { mejorarse notablemente } \\
\text { los rasgos que definen } \\
\text { su orientación al logro }\end{array}$ \\
\hline
\end{tabular}

13 $^{\text {th }}$ LACCEI Annual International Conference: "Engineering Education Facing the Grand Challenges, What Are We Doing?" July 29-31, 2015, Santo Domingo, Dominican Republic 


\begin{tabular}{|l|l|l|}
\hline 1 & $1 \%-25 \%$ & $\begin{array}{l}\text { No se cumple. Le falta } \\
\text { interés y voluntad para } \\
\text { desarrollar habilidades } \\
\text { de perseverancia y } \\
\end{array}$ \\
& $\begin{array}{l}\text { orientación al logro. } \\
\text { Presenta r más } \\
\text { debilidades } \\
\text { fortalezas que }\end{array}$ \\
\hline
\end{tabular}

Una vez aplicadas las encuestas se determinó el nivel de cumplimiento de cada una de las características y así se determinó las fortalezas de los estudiosos del programa:

TABLA VI

RESULTADOS: CARACTERÍSTICA ORIENTACIÓN A RESULTADOS

\begin{tabular}{|l|c|c|}
\hline \multicolumn{1}{|c|}{ Característica Orientación a Resultados } & \multicolumn{2}{c|}{ Resultados 2015 } \\
\cline { 2 - 4 } & \% Total & $\begin{array}{c}\text { Grado de } \\
\text { Cumplimiento }\end{array}$ \\
\hline $\begin{array}{l}\text { ¿Te mantienes firme y calmado en situaciones } \\
\text { de trabajo bajo presión y/o oposición? }\end{array}$ & 82 & $\begin{array}{c}\text { Se cumple en alto } \\
\text { grado }\end{array}$ \\
\hline $\begin{array}{l}\text { ¿Mantienes el norte en cualquier situación de } \\
\text { cambio? }\end{array}$ & 87 & $\begin{array}{c}\text { Se cumple en alto } \\
\text { grado }\end{array}$ \\
\hline $\begin{array}{l}\text { ¿Persistes en resolver un problema hasta } \\
\text { comprobar que quede resuelto? }\end{array}$ & 86 & $\begin{array}{c}\text { Se cumple en alto } \\
\text { grado }\end{array}$ \\
\hline $\begin{array}{l}\text { ¿Persistes en cualquier asunto hasta } \\
\text { comprobar que se ha alcanzado el objetivo en } \\
\text { un período razonable? }\end{array}$ & 83 & $\begin{array}{c}\text { Se cumple en alto } \\
\text { grado }\end{array}$ \\
\hline $\begin{array}{l}\text { ¿Sientes necesidad de alcanzar un objetivo } \\
\text { con éxito? }\end{array}$ & 93 & $\begin{array}{c}\text { Se cumple } \\
\text { plenamente }\end{array}$ \\
\hline $\begin{array}{l}\text { ¿Eres constante con las iniciativas que } \\
\text { emprendes? }\end{array}$ & 87 & $\begin{array}{c}\text { Se cumple en alto } \\
\text { grado }\end{array}$ \\
\hline
\end{tabular}

De acuerdo al análisis anterior, se concluye que la característica orientación a resultados se cumple en alto grado con un promedio de $86 \%$, denotando la fortaleza que posee el estudioso del programa de Ingeniería Industrial y que deben tender al mejoramiento pleno, orientadas a impactar profundamente su propio desafío para la consecución de objetivos de interés propio y general.

\section{DISCUSIÓN}

Teniendo en cuenta los resultados del análisis de cada una de las características investigadas, se puede afirmar que existe coherencia entre las dimensiones planteadas para describir un perfil emprendedor: liderazgo, creatividad y orientación a resultados, las cuales hacen parte de las características reconocidas como parte del perfil emprendedor por los estudiantes de la Universidad Manuela Beltrán.

De las variables analizadas del conjunto de características estudiadas, siete de ellas presentan cumplimiento pleno, que equivale al $41 \%$ de la evaluación total.
El emprendimiento está relacionado por excelencia a nuevas y mejores formas de analizar y resolver necesidades en el contexto empresarial y al desarrollo de nuevos productos y servicios con innovación, lo que se integra de manera significativa y positiva con este resultado de consistencia frente al alto grado en que se cumple la característica de creatividad en los estudiantes de Ingeniería Industrial.

En general estos resultados obtenidos, además de demostrar la confiabilidad del instrumento y de la metodología empleada, presentan una implicación teórica importante: son la primera manifestación concreta de las percepciones y la experiencia del equipo interdisciplinario del Centro de Emprendimiento CREAR y la Dirección del Programa de Ingeniería Industrial respecto a las expectativas de comportamiento de un emprendedor, la cual puede llegar a relacionarse con varios de los postulados teóricos en la literatura de emprendimiento.

Estas primeras tres características analizadas en esta investigación, resaltan el posicionamiento del emprendedor como un individuo enérgico, emocionalmente autorreconocido y aceptado, con capacidad de liderar grupos y desarrollar planes creativos para la ejecución exitosa de una tarea, por encima incluso de las características que componen la motivación de logro, y con un marcado sentido de orientación al logro.

\section{CONCLUSIONES}

Considerando la importancia de esta investigación para la caracterización del perfil emprendedor de los estudiantes del programa de Ingeniería Industrial de la Universidad Manuela Beltrán, el resultado de la misma puede resumirse en:

Considerando que las instituciones educativas son el pilar fundamental de desarrollo, deberán ser protagónicas el fomento de políticas, programas, estrategias, acciones, etc., que fortalezcan el espíritu emprendedor en sus diferentes grupos de interés, enmarcado en las herramientas ofrecidas por la Ley de Emprendimiento del país.

$>$ El mejoramiento de la calidad de vida de nuestros estudiantes del programa de ingeniería industrial, está ligado a la posibilidad de emprender negocios que garanticen la sostenibilidad propia y de su grupo familiar

$>$ Las instituciones educativas deben extender su proyección social al desarrollo de programas de asesorías, consultorías, etc., para la creación y formalización de unidades productivas de negocios, que nacen de la necesidad de independencia de sus estudiantes.

Dado que la relación de la estrategia de emprendimiento universitario está articulada al

13 $^{\text {th }}$ LACCEI Annual International Conference: "Engineering Education Facing the Grand Challenges, What Are We Doing?" July 29-31, 2015, Santo Domingo, Dominican Republic 
desarrollo local y regional de los pueblos, deberá asimilarse desde la academia, en su formación emprendedora y empresarial.

$>$ Se requiere continuar con el fortalecimiento de las habilidades y competencias de nuestros estudiosos, esencialmente los rasgos para formar la personalidad del emprendedor, teniendo como soporte fundamental el saber hacer y el conocimiento del entorno; se elaborarán proyectos y planes de empresa sostenibles, generando empleo y trabajo calificado con profesionales de alta competencia

\section{REFERENCIAS}

[1] SENA (1993). Programas de asesoría a la micro, pequeña y mediana empresa; y, programa de formación a creadores de empresa. Revista Proposiciones, (23), 290-306. http://www.sena.edu.co/acerca-delsena/quienes-somos/Paginas/Quienes-Somos.aspx

[2] Congreso de la República de Colombia (1990). Ley 29 de 1990, por la cual se dictaron disposiciones para el fomento de la investigación científica y el desarrollo tecnológico.

[3] Informe Misión de Sabios; Colombia al filo de la Oportunidad http://www.icesi.edu.co/investigaciones_publicaciones/images/pdf/colom bia filo de la oportunidad.pdf

[4] Congreso de la República de Colombia (1996). Ley 344 de 1996, por la cual se dictan normas tendientes a la racionalización del gasto público, se conceden unas facultades extraordinarias y se expiden otras disposiciones

[5] Congreso de la República de Colombia (1997). Ley 375 de 1997, por la cual se crea la ley de la juventud y se dictan otras disposiciones.

[6] Congreso de la República de Colombia (2000), Ley 590 de 2000, Impulsar el desarrollo de la micro, pequeña y mediana empresa colombiana.

[7] Congreso de la República de Colombia (2002). Ley 789 de 2002, Creación FONDO EMPRENDER

[8] Departamento Nacional de Planeación (2008). Documento Conpes: 3597, 3424, 3439, 3484,3527. Bogotá: DNP.

[9] Ministerio de Educación Nacional (2012), La cultura del emprendimiento en los establecimientos educativos, P.10

[10] Congreso de la República de Colombia (2004). Ley 905 de 2004

[11] Congreso de la República de Colombia (2006). Ley 1014 de 2006, de fomento a la cultura del emprendimiento

[12] Congreso de la República de Colombia (2008). Ley 1151 de 2007.

[13] Congreso de la República de Colombia (2008), Ley 1258 de 2008

[14] Departamento Nacional de Planeación (2008). Documento Conpes 3597. Bogotá: DNP.

[15] Departamento Nacional de Planeación (2008). Documento Conpes 3424. Bogotá: DNP.

[16] Departamento Nacional de Planeación (2008). Documento Conpes 3439. Bogotá: DNP.

[17] Departamento Nacional de Planeación (2008). Documento Conpes 3484. Bogotá: DNP.

[18] Departamento Nacional de Planeación (2008). Documento Conpes 3527. Bogotá: DNP.

[19] Ministerio de Educación Nacional (2012), La cultura del emprendimiento en los establecimientos educativos, P.10

[20] Páez, Diana (2003). Acercamiento a las características del universitario emprendedor en la unidad emprendimiento empresarial de la Universidad Nacional de Colombia, Revista Escuela de Administración de Negocios No.71, Julio-Diciembre 2007. EAN

[21] Csickzentmihalyi (1998) Creatividad. Barcelona: Paidós.

[22] Cooper, A., Ramachandran, M., Schoorman, D. (1997). Entrepreneurial time allocation: antecedents and performance implications. Frontiers of Entrepreneurship Research
[23] García, J., et al (2005) Análisis de actitudes emprendedoras en estudiantes universitarios y empresarios. Evidencia de validez de un instrumento Iberpsicología Anales de la Revista de psicología general y $\begin{array}{llllll}\text { aplicada. } & \text { Lisboa: 2005: } & \text { Vol. } & 10 . & \text { No } & 8 .\end{array}$ http://www.fedap.es/IberPsicologia/iberpsi10/congreso lisboa/garcia2/gar cia2.htm.

[24] Hisrich, R., et al (2007) Entrepreneurship research and practice. A call to action for psychology. Journal American Psychological Association. Vol. 62 No. 65 Pág 575-589.

[25] Ley 1014. (26/01/2006). De fomento a la cultura del emprendimiento. Grupo Integrado de Emprendimiento, Empresarismo y Fondo Emprender SENA

[26] Spencer, L. \& Spencer, S. "Competence at Work". Jhon Wiley \& Sons, Toronto, 1993.

[27] Zhao H., Seibert S., (2006) The big fi ve personality dimensions and entrepreneurial status: a meta analytical review. Journal of apllied psychology VOL.91 No 2 Pág. 259-271.

13 ${ }^{\text {th }}$ LACCEI Annual International Conference: "Engineering Education Facing the Grand Challenges, What Are We Doing?" July 29-31, 2015, Santo Domingo, Dominican Republic 